\title{
Norois
}

Environnement, aménagement, société

$215 \mid 2010 / 2$

Impacts morphogéniques des tempêtes

\section{Tempêtes, rythme de fonctionnement d'une cellule sédimentaire et « espace d'accueil » : exemple sur l'Anse du Verger, Bretagne Nord}

Storms, rhythms of a sediment cell and accommodation space: an exemple in northern Brittany, the Anse du Verger

Hervé Regnauld, Haya Mahmoud, Johan Oswald, Olivier Planchon et Jonathan Musereau

\section{OpenEdition \\ Journals}

Édition électronique

URL : https://journals.openedition.org/norois/3279

DOI : $10.4000 /$ norois. 3279

ISBN : 978-2-7535-1563-5

ISSN : 1760-8546

Éditeur

Presses universitaires de Rennes

Édition imprimée

Date de publication : 30 septembre 2010

Pagination : 133-146

ISBN : 978-2-7535-1164-4

ISSN : 0029-182X

Référence électronique

Hervé Regnauld, Haya Mahmoud, Johan Oswald, Olivier Planchon et Jonathan Musereau, «Tempêtes, rythme de fonctionnement d'une cellule sédimentaire et « espace d'accueil » : exemple sur l'Anse du Verger, Bretagne Nord », Norois [En ligne], 215 | 2010/2, mis en ligne le 01 septembre 2012, consulté le 06 décembre 2021. URL : http://journals.openedition.org/norois/3279 ; DOI : https://doi.org/10.4000/ norois.3279

(c) Tous droits réservés 


\title{
TEMPÊTES, RYTHME DE FONCTIONNEMENT \\ D'UNE CELLULE SÉDiMENTAIRE ET « ESPACE D'ACCUEIL » : \\ exemple sur l’Anse du Verger, Bretagne Nord
}

\author{
Hervé Regnauld, Haya Mahmoud, Johan Oswald, \\ Olivier Planchon, Jonathan Musereau \\ Laboratoire Costel - UMR 6554 LETG CNRS \\ (Université de Rennes 2 et Université Européenne de Bretagne), \\ Place H.-Le-Moal - 35043 Rennes cedex, France \\ herve.regnauld@univ-rennes2.fr,haya.mahmoud@univ-rennes2.fr, johan.oszwald@univ-rennes2.fr, \\ olivier.planchon@univ-rennes2.fr, jonathan.musereau@club-internet.fr
}

\section{RÉSUMÉ}

L'évolution du littoral est parfois pensée comme le résultat de situations météorologiques dominantes, parfois comme le produit des effets d'événement extrêmes tels que les très fortes tempêtes. Sur la côte Nord de la Bretagne, un site étudié depuis 20 ans a évolué sous le contrôle de politiques d'aménagement et selon le forçage de longues périodes de temps calmes et de trois très fortes tempêtes. Ce travail tente d'assigner à chacun de ces trois processus une part de responsabilité dans les tendances d'évolution constatées. Leur rôle est hautement variable dans le temps et dans l'espace. Il devient alors très difficile de penser l'évolution selon un modèle simple de contrôle, forçage et résilience, parce que ces trois notions n'ont pas nécessairement le même sens selon les lieux et les formes qu'elles concernent. Cependant les tempêtes apparaissent déterminantes pour une large portion du littoral étudié.

\section{MotS CLÉ : tempête - espace d'accueil sédimentaire - évolution - Bretagne}

\section{ABSTRACT \\ Storms, rhythms of a sediment cell and accommodation space: an exemple in northern Brittany, the Anse du Verger}

Coastal evolution is sometimes said to be forced by storms, sometimes by average meteorological conditions. The exact role of very strong storms is therefore a widely debated issue. On the northern coast of Brittany (Western France) a site has been surveyed for the last 20 years and has evolved under the control of human management and the forcings of long periods of calm weather and of three large storms. This paper tries to asses the weight of each of these agents on coastal evolution. Their respective efficiency is highly variable in time and in space, thus making it very difficult to understand coastal behaviour with a simple conceptual model of forcings, controls and resilience These notions do not have the same meaning depending on where they are applied but storms appear as the main forcing on a large extent of the studied coastline.

KEY WORDS : storm - accommodation space - evolution - Brittany 
Une question largement débattue en géomorphologie littorale est celle des forçages sur les rythmes d'évolution du trait de côte des accumulations sédimentaires. Le fait que de temps à autres de fortes tempêtes produisent de fortes érosions incite parfois à penser que l'évolution est forcée par des événements intenses et que c'est en étudiant les variations de leurs fréquences que l'on peut le mieux expliquer les vitesses de recul d'un littoral précis (Cooper, 2002 ; Musereau et al., 2007). Cependant de nombreux travaux montrent qu'un littoral s'aligne souvent face aux houles dominantes alors que les directions de houles de tempêtes les plus fortes ne sont pas toujours celles des houles dominantes (voir la synthèse de J. Dubois, 1995, puis celles de P. Roy et P. Cowell, 1996 et J. Dodd et al., 2003). Il y a donc débat. L'importance des tempêtes est une idée fondée sur de nombreuses observations. Elle a été particulièrement développée dans quatre publications de synthèse, pionnières et originales (Carter et Orford, 1993; Forbes et al., 1995; Anthony et al., 1996; Orford et al., 2002). Ces travaux ont valorisé des concepts nouveaux en géomorphologie, dont celui d'accommodation space qui n'était jusqu'alors employé qu'en stratigraphie séquentielle (Jervey, 1988). L'originalité de cette position épistémologique est de penser le comportement du littoral au travers d'un couple dynamique, la résilience et l'accommodation ou espace d'accueil. Schématiquement il s'agit de considérer que, pour les littoraux constitués par des accumulations de sédiment (des galets en l'occurrence), les forçages principaux sont d'ordre météo-marin, tandis que les contrôles dominants sont spatiaux. Le corps sédimentaire est capable de se déformer et de se déplacer pour trouver la configuration qui lui permet de subsister face un événement à haute énergie. Ce changement de forme lui permet de dissiper l'énergie reçue et est interprété comme une forme d'auto organisation (Orford et al., 2002). La capacité à se déformer sans se rompre est définie comme la résilience, le déplacement est contrôlé par l'espace disponible (accommodation space) pour les overwash et rollover. Avec une telle approche on peut facilement expliquer qu'une tempête qui fait, ponctuellement, reculer une partie du littoral (rollover par exemple) peut aussi, en un autre lieu, construire le littoral, voire augmenter sa hauteur par phénomène d'overtopping. Il est important de penser les deux phénomènes en même temps.

À l'évidence une vision aussi contre intuitive des effets des tempêtes n'a pas toujours été bien accueillie. Elle permet cependant de bien comprendre les effets d'une tempête et, suggérant de prendre du recul sur le coté spectaculaire d'une érosion ponctuelle, elle incite à chercher les sites où la tempête a non pas érodé mais construit. Le cadre idéal pour une telle démarche est celui d'une cellule sédimentaire (Bray et al., 1995), dont on peut postuler que les sites sources vont reculer sous l'effet d'une tempête et que les sites puits vont, en conséquence et peu après, s'engraisser. Pour étudier dans ce contexte le rôle des tempêtes, un site intéressant serait celui pour lequel le suivi de telles cellules sur le moyen terme (10,20 ans) peut permettre de définir des vitesses moyennes et des tendances, et où un suivi de chaque événement intense peut permettre de préciser son rôle exact, ses conséquences et donc son importance relative par rapport aux conditions moyennes. Sur le littoral de Bretagne nord, entre Saint-Malo et Cancale, plusieurs anses fonctionnent en apparence comme de petites cellules sédimentaires à l'échelle locale. L'une d'entre elles, l'Anse du Verger (fig. 1) est suivie depuis 1990 et n'a subi durant ce temps que trois tempêtes «fortes », en 1991, 1995 et 2008. Il est donc possible de décrire son évolution en terme de tendance comme en terme d'événement. L’idée générale est de comparer le recul du site « source », l'engraissement du site «puits » et de déterminer s'il s'agit d'un rythme dépendant des tendances météo-marines modales ou des événements intenses comme les tempêtes.

\section{Présentation du site}

L'Anse du Verger correspond à un cordon de fond de baie. Les pointes rocheuses qui l'encadrent sont tapissées de dépôts périglaciaires d'épaisseur variable et le cordon dunaire enferme un marais d'eau douce. En 2008, avant la tempête du 10 mars, le site comprend plusieurs unités morphologiques. La photo de la figure 1 permet de distinguer, à côté du parking, une dune aménagée indiquée par A sur la photo (avec des ganivelles et des chemins) et un marais d'eau douce 
(cartouche 3 sur la figure). Plus à l'est se situe une falaise taillée dans les dépôts périglaciaires (désignée par B) puis une falaise dans de la roche en place, des migmatites (désignée par C). La plage s'étend devant les unités $\mathrm{A}, \mathrm{B}$ et $\mathrm{C}$.

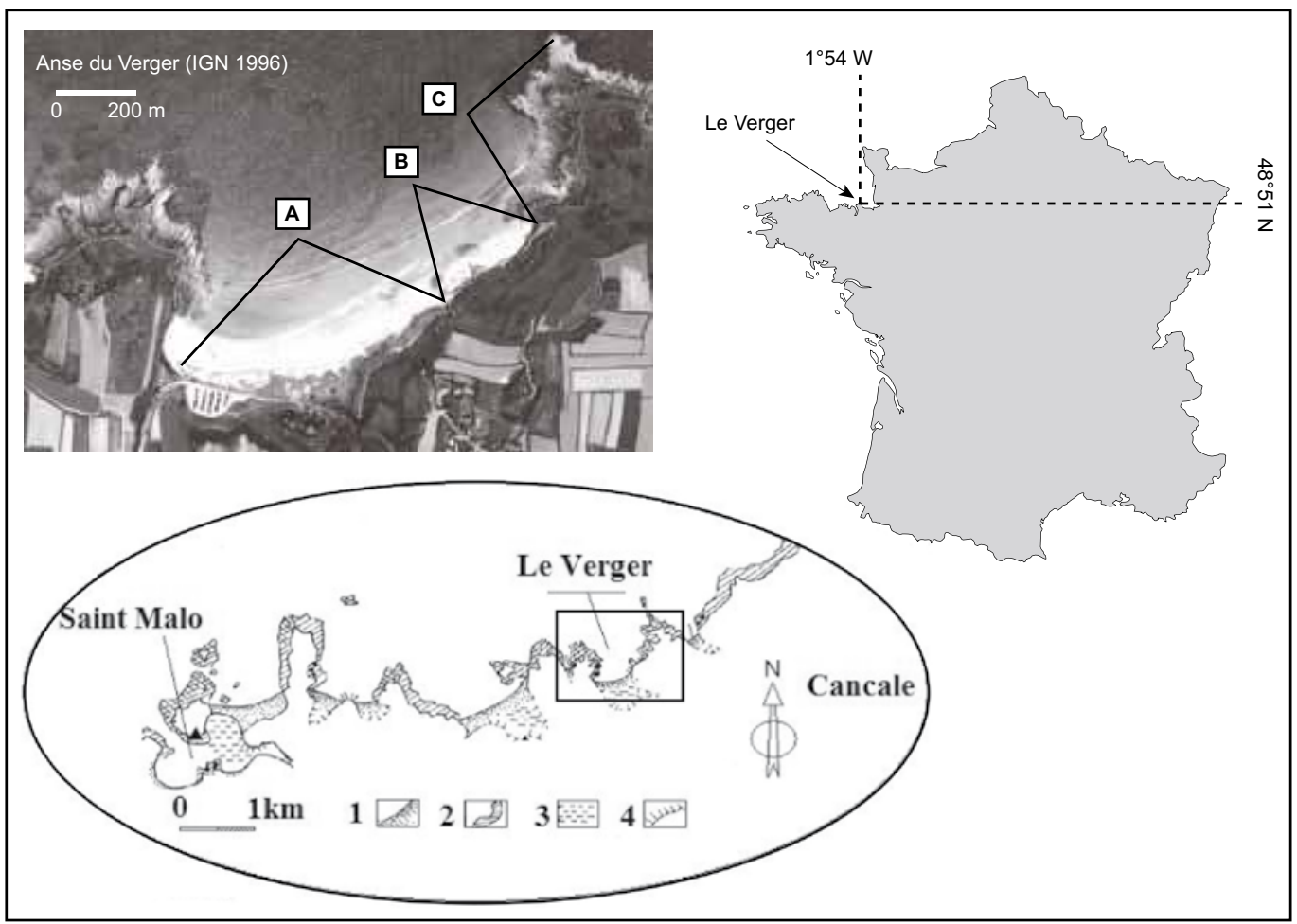

Figure 1 : Localisation du site étudié

1 : plage. 2 : plate-forme d'abrasion. 3 : marais. 4 : falaise rocheuse. Sur la photo A désigne l'ensemble plage/dune, B l'ensemble plage/falaise dans le matériel périglaciaire, C l'ensemble plage/platier/falaise en roche dure.

Location of the field site

1: beach. 2: wave cut platform. 3: marsh. 4: cliff. On the air photo A is the beach/dune unit, B the beach/soft rock cliff unit, $\mathrm{C}$ is the beach/abrasion platform/hard rock cliff unit.

La falaise dans le matériel périglaciaire présente une coupe dont les différentes unités varient le long du linéaire. Là où toutes les unités sont présentes, elle va probablement de l'Eémien à l'actuel (Regnauld et al., 2003). À la base, sur une plate-forme d'abrasion fossile, se trouve une plage de galets (attribuée à l'Eémien mais sans datation absolue) puis une dune (datée à 90 ky OSL) et un head indifférencié (fig. 2). Il est surplombé par un loess pléniglaciaire puis par une dune Holocène (6 ky OSL et divers âges $14^{\mathrm{C}}$ entre 6000 et 2000 BP). Le sommet est un sol historique daté par des artéfacts celtiques (Cocaign et al., 1996) recouvert par une dune sub-actuelle, dont l'âge de mise en place est inconnu. On y a retrouvé des artefacts datant du Premier Empire. Le cordon dunaire est daté de environ 4600BP (par un kjokkenmodding, dépôt de cuisine néolithique recouvert d'un dépôt de tempête (Regnauld et al., 1995). Le marais qu'elle protège avait commencé à se mettre en place vers 8000 BP (Regnauld et al., 1996). À l'occasion de la tempête de 1991, un site paléolithique a été découvert dans la partie de la falaise qui avait reculé à cette occasion (partie B sur la fig. 1).

Certains de ces artefacts ont été repérés sur la plage et au cours de jours suivants, d'autres ont été observés en divers lieux de l'Anse, puis au pied de la dune (dans la partie A, sur la photo de la 
fig. 1). L'idée que la falaise pouvait être un site « source » et que le cordon était un site "puits » est naturellement venue de ces observations. Elle a été qualitativement confirmée par des observations en plongée (Regnauld et al., 1997).

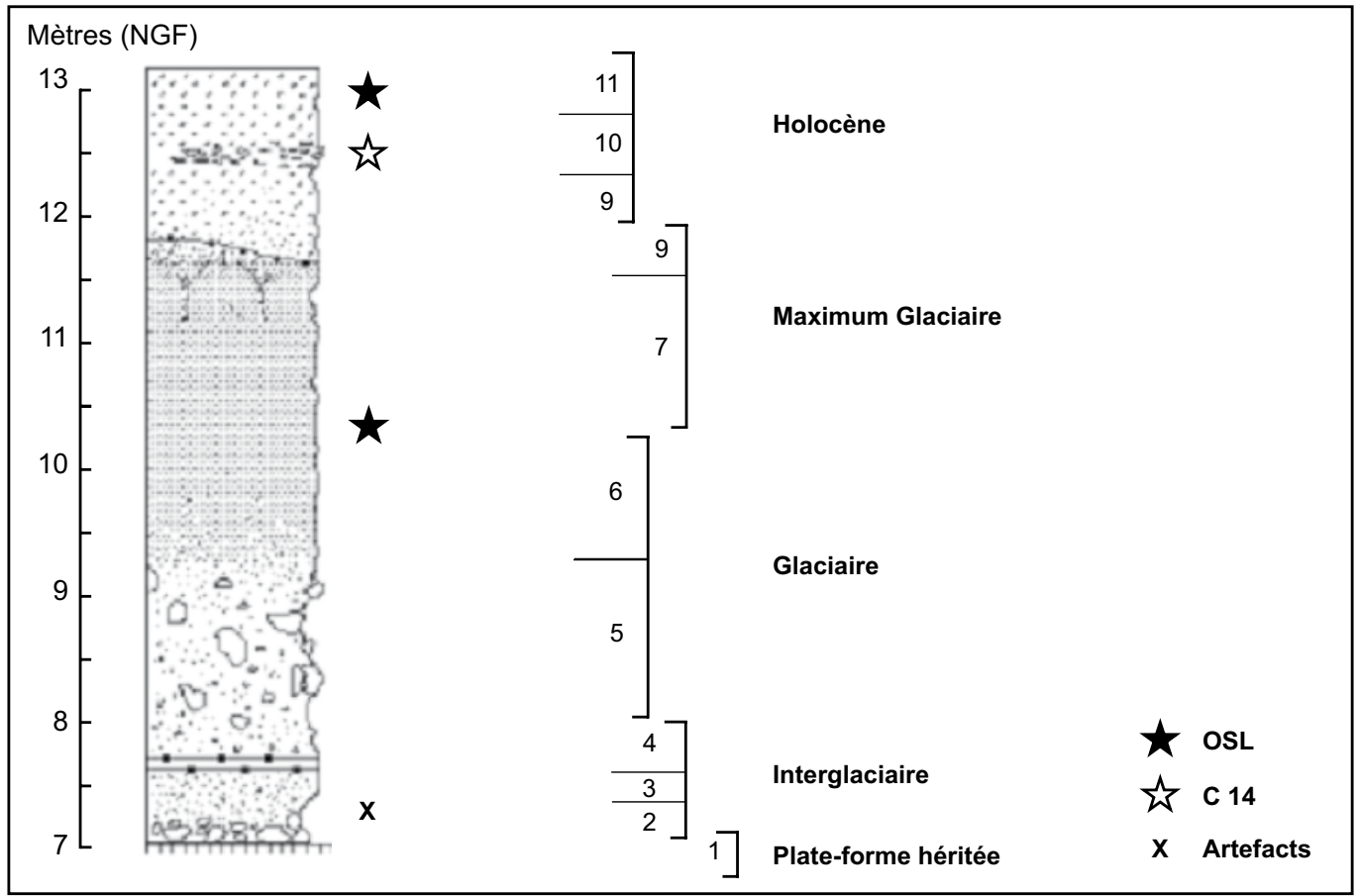

Figure 2: Coupe levée en 2000, aujourd'hui disparue à cause du recul (publiée dans Regnauld et al., 2003)

1 : Plate-forme d'abrasion héritée. 2 : Galets attribués à l'Eémien. 3 : Dune datée à $90 \mathrm{k}$ an OSL. 4 : Paléo sol. 5 : Head; 6 : Loess. 7 : Loess avec cryoturbations. 8 : Paléo sol. 9 : Sable dunaire; 10 : Kjokkenmoddings néolithiques; 11 : Dune sub-actuelle. Les points mesurés pour étudier le recul de ce type de falaise sont d'une part le sommet de la dune perchée (sommet de 11), d'autre part le contact entre les galets et la plate forme d'abrasion (entre 1 et 2). Cette coupe sert de modèle pour le type C de la figure 7.

Cross section, surveyed in 2000, gone today with cliff retreat (published in Regnauld et al., 2003) 1: Inherited abrasion platform; 2: Eemian pebbles; 3: Dune (90 ky OSL); 4: Palaeo soil; 5: Head; 6: Loess; 7: Loess with cryoturbations; 8: Palaeo soil; 9: Dune sand; 10 : Neolithic knokkenmodding; 11: Dune (+/present). To monitor the retreat of this cross section, the surveyed points are the top of dune ( $\left.n^{\circ} 11\right)$ and the contact between the pebbles and the inherited platform. This cross section is the model for type $C$ in figure 7.

\section{Méthodes}

Les méthodes employées pour cette étude sont classiques. Depuis 1990, des profils de plage sont levés régulièrement au DGPS (environ un par mois en hiver, environ un tous les trois mois en été) et calés à partir d'une borne géodésique installés par l'IGN sur un des rochers affleurant sur l'estran. Leur marge d'erreur verticale est de l'ordre du décimètre. Cette valeur semble élevée mais il s'agit d'un écart maximal. Le problème vient de la nature molle du substrat sableux, lorsqu'il est saturé en eau après les hautes mers de vives eaux. Le simple fait de marcher dessus en change la topographie de quelques centimètres. L'erreur maximale a été considérée en retenant la valeur la plus élevée que nous ayons rencontrée. Elle correspond aux mesures de mars 2008 (juste après la tempête), quand le sédiment du pied de falaise avait le comportement le plus plastique. 
Des profils sont également effectués sur le cordon dunaire et sur la falaise, pour construire des coupes. En ce qui concerne la falaise, son sommet est facile à identifier mais sa base pose problème : elle est plus ou moins enfouie dans le sable selon l'état d'engraissement de la plage. Dans la mesure du possible, la base a été prise au contact entre les dépôts paléo- littoraux ou périglaciaires (dont la géométrie et la nature varient le long de la falaise) et le socle sub-affleurant. Ce contact peut être dû à des unités sédimentaires in situ (entre les unités 1 et 2 de la figure 2 par exemple) ou à du matériel effondré. Cette opération de mesure est renouvelée tous les 10 mètres environ, voire à des intervalles plus courts si un trait morphologique du profil le nécessite. La figure 2 donne un exemple d'une telle coupe, levée en 2000 et aujourd'hui disparue suite au recul de la falaise. Une carte schématique où figure l'emplacement de la base et du sommet de la falaise (séries de points) est également produite afin de comparer leurs évolutions respectives.

Enfin, des prélèvements sédimentaires sont effectués dans les différentes unités qui apparaissent en coupe dans la falaise, ainsi qu'en divers points du cordon dunaire, de la plage et de la plage sous-marine. Des analyses granulométriques et morphoscopiques sont ensuite réalisées. Ces échantillonnages ont eu lieu deux fois par an entre 1990 et 1996. Ils donnaient toujours des résultats très similaires et ont donc été abandonnés. Ils ont été repris en 2008, après la tempête.

Les grandes lignes de l'histoire du site durant le dernier demi-siècle ont été reconstituées à partir de documents transmis par la DDE, le Département d'Ille-et-Vilaine, le Conservatoire du Littoral et à l'aide d'une chronique de photos aériennes. Les clichés dont nous disposons datent de 1948, 1952, 1977, 1980, 1982, 1996 et 2006. Ils ont été scannés et géoréférencés selon des méthodes classiques avec le logiciel ENVI 4.4 (Pian et al., 2008). La photographie aérienne la plus récente est géoréférencée à partir de la BD ortho éditée par l'IGN (France zone 2 étendue, NTF). Pour limiter les distorsions, 32 points d'amers ont été utilisés afin d'éviter les erreurs engendrées par l'anisotropie spatiale (RMS allant de 1.9 à 3.1). Le sommet de la falaise a été retenu comme indicateur morphologique pour étudier la mobilité du trait de côte. La marge d'erreur des images obtenues est relativement variable, de 2 à 5 mètres, et comparables à ce qui est avancé dans la littérature (Robin, 2002). Pour des questions de contraste, il est parfois très difficile de situer le sommet de la falaise, en particulier si celui-ci est couvert de végétation. En revanche, quand le sommet de falaise est dénudé par le piétinement, il apparaît comme du sol très clair et se différencie très bien du versant sombre ou de son ombre. Là encore nous avons retenu une erreur de $5 \mathrm{~m}$, la plus importante déviation au sol que nous puissions estimer.

\section{Effets de la tempête et formes de recul}

Durant la tempête du 10 mars 2008, décrite par ailleurs dans ce numéro de Norois (Cariolet et al., ce volume), les conditions locales au Verger ont été marquées par des houles d'amplitude estimée à la côte à $6 \mathrm{~m}$. La comparaison des levés effectués avant ( ${ }^{\mathrm{er}}$ mars 2008) et après la tempête (12 mars 2008) fait apparaître que le recul de la falaise taillée dans le périglaciaire (en B sur la fig. 1) est très important mais très inégal suivant les lieux et les parties de la falaise. Dans l'ensemble le recul moyen est largement supérieur au mètre, ce qui est comparable aux reculs engendrés par les tempêtes de 1990 et 1996. La figure 3 représente la position des points témoins de la position du pied de la falaise au contact de la base du matériel périglaciaire avec la roche en place. Ils reculent partout sauf dans la partie centrale où de nombreux éboulements et effondrements fossilisent l'emplacement pré-tempête de la base du profil. Ce fait est discuté plus loin.

Le sommet de la falaise (fig. 3) recule également, de 1 à 4 mètres. Le lendemain de la tempête de nombreux pans de falaise sont encore tenus par la végétation : des touffes d'ajoncs fonctionnent en chablis. Il y a très peu de débris au pied (hormis dans la partie centrale, signalée au dessus). Au cours des jours suivants tous ces chablis tombent, le recul du sommet s'accentue et les dépôts de pied de falaise deviennent plus importants. Lors du levé du 12 mars, le recul mesuré est donc l'addition de celui qui a eu lieu durant la tempête elle-même et de celui qui a été provoqué par 
les effondrements postérieurs induits par les chablis. De tels effondrements ont continué durant toute l'année et bien qu'aucune tempête forte n'ait atteint le site, la falaise continue à reculer : le 20 janvier 2009, son sommet est partout en retrait par rapport à mars 2008.

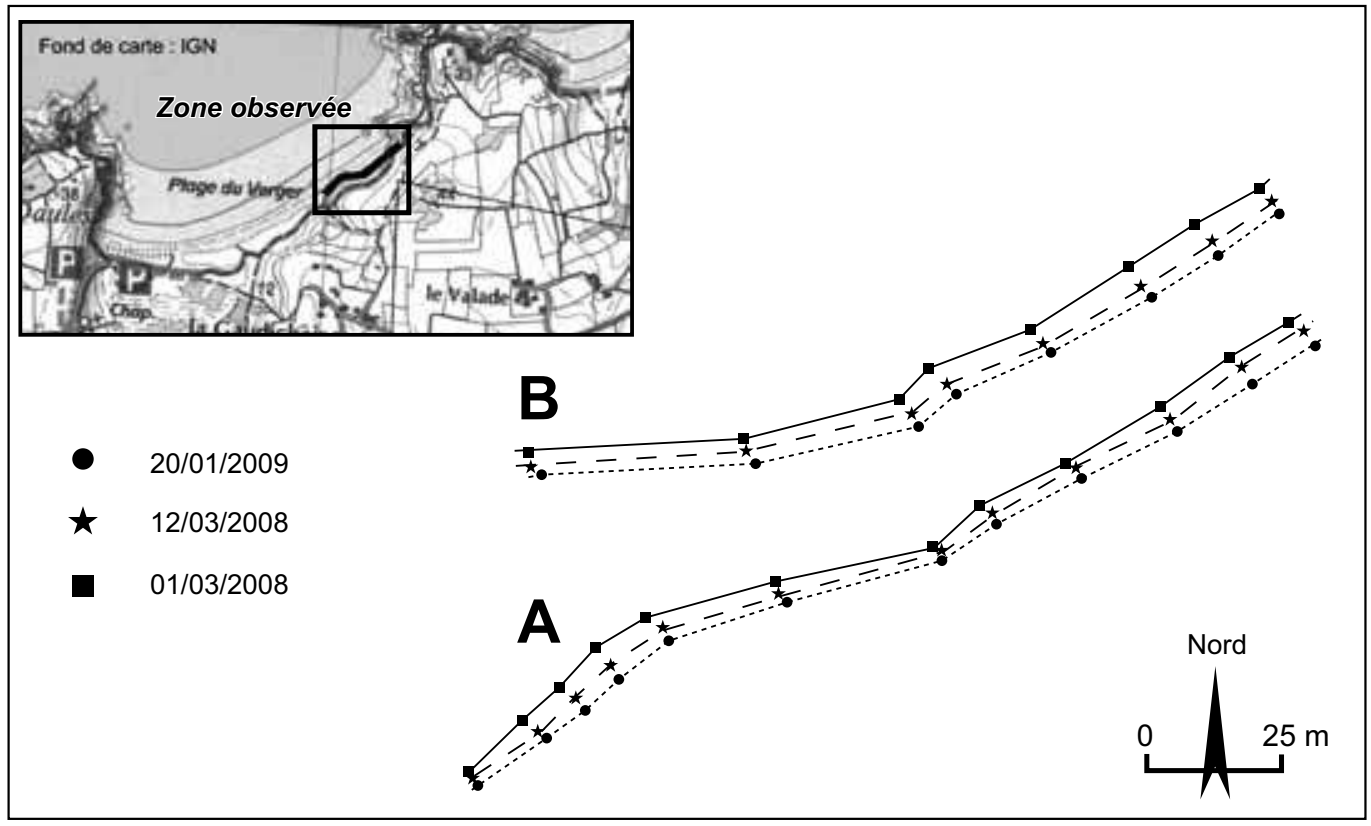

Figure 3 : Évolution du sommet de la falaise (A) et de son pied (B) entre le $1^{\text {er }}$ mars 2008 , le 12 mars 2008 et le 20 janvier 2009, marge d'erreur à $0,1 \mathrm{~m}$. Explications dans le texte.

Evolution of cliff top (A) and foot (B) between 3-1-2008, 3-12- 2008 and 1-20-2009, error margin at $0,1 \mathrm{~m}$. Comments in the text.

Le long du cordon, la tempête a arraché toutes les ganivelles, et enlevé suffisamment de sable pour faire apparaître des ganivelles anciennes et enfouies. L'ensemble du massif a perdu 1,5 m sur toute sa longueur, de façon relativement régulière. Il a été alors taillé en micro falaise de 1 à $1,5 \mathrm{~m}$ de hauteur... Lors de la tempête, les vitesses de vent sur place (avec les réserves signalées auparavant) ont atteint de 4 à $7 \mathrm{~m} . \mathrm{s}^{-1}$ au sol $($ à $10 \mathrm{~cm})$ et de 13 à $26 \mathrm{~m} . \mathrm{s}^{-1}$ à deux mètres d'altitude.

Les reculs produits par la tempête ont induit des transferts de matériel. Entre 1991 et 1996 des échantillonnages avaient été systématiquement effectués dans le sable le long du pied de la falaise, sur la plage et la plage sous-marine ainsi que dans le cordon dunaire. Les analyses granulométriques et morphoscopiques (Regnauld et al., 1992, 1995) ont permis d'établir que le recul de la falaise fournissait du matériel à la plage sous-marine et que celui-ci était ensuite en partie recyclé sur le cordon, sous la forme de dépôts de tempêtes (washover) venant recouvrir des dépôts éoliens. Les valeurs moyennes pour les courbes granulométriques de la plage sous-marine, de la plage intertidale et du cordon sont présentées sur la figure 4.

Apres la tempête du 10 mars 2008, de nouveaux échantillonnages ont été réalisés. Au pied de la falaise, le sable de la plage est considérablement enrichi en fraction grossière (2 mm et plus) et appauvri en sables moyens de 1 à $0,2 \mathrm{~mm}$. À l'inverse la plage sous-marine s'est considérablement enrichie dans cet intervalle (1-0,250 mm) et la fraction fine s'est appauvrie. 
Figure 4 : Granulométrie des échantillons du pied de falaise et de la plage sous-marine, avant et après la tempête

Grain size for the cliff foot and for the sub marine beach before and after the storm
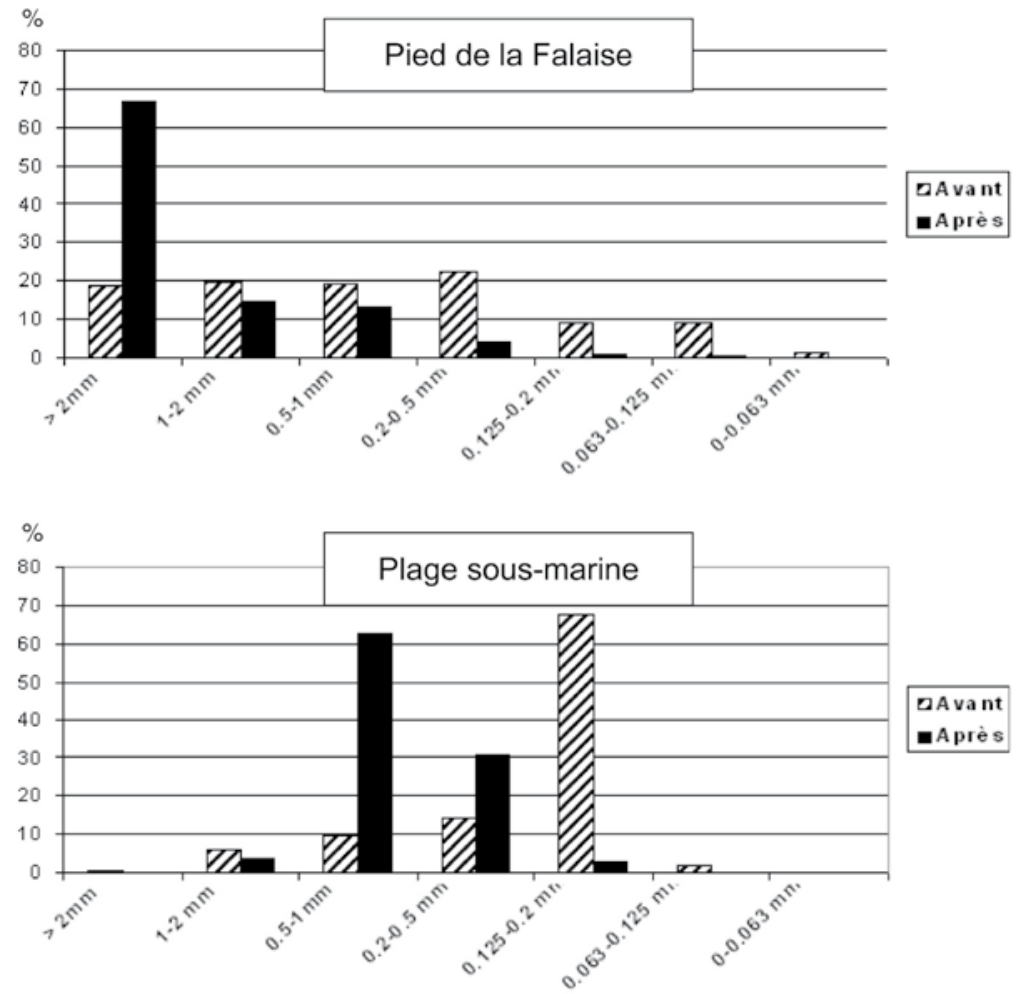

\section{Tempêtes et évolution des formes sur 60 ans}

Le recul produit par la tempête du 10 mars 2008 sur le site du Verger peut être qualifié de « spectaculaire ». Cet événement morphogénique est-il pour autant exceptionnel sur ce site? Ses effets sont-ils comparables à ce que l'évolution habituelle du littoral produit ou cette tempête a-telle été capable de produire des effets inattendus, voire contraires à son évolution tendancielle? Pour répondre à ces questions, il faut reconstituer la morphodynamique récente du site.

En 1948, le Verger est un site très peu touristique qui ne dispose d'aucun aménagement (ni camping, ni voie carrossable d'accès). Toutes les parcelles alentours sont cultivées et souvent encloses. L'accès pédestre est difficile. En 1952, la partie ouest commence à être érodée, probablement à la suite d'une surfréquentation (fig. 5). Dans les années 1960-1962, de grandes quantités de sable sont prélevées dans le cordon dunaire pour fabriquer le béton de l'usine marémotrice de la Rance. Le cordon dunaire ne joue plus son rôle de barrière et le marais peut parfois être atteint par des lames d'eau salée. Le site devient accessible : les voitures suivent les chemins tracés par les camions. De 1960 à 1985, le site est donc très fréquenté, appauvri en sédiment et l'érosion y est très forte. Le littoral recule beaucoup, au point que l'État (DDE) et le département (Bureau des Espaces Naturels Sensibles) s'en inquiètent. À partir de 1985, il est mis en réserve partielle et une politique de réaménagement est mise en place sous l'impulsion du Conservatoire du Littoral, qui devient ensuite propriétaire. Le cordon est mis en réserve et la falaise est laissée à son recul : après la tempête de 1995, le chemin littoral est recréé trente à quarante mètres plus à l'intérieur.

La figure 6 donne une vue schématique des principales étapes de cette évolution. Entre 1948 et 1952, il n'y a pas de recul visible. À cette époque, il n'y a pas de falaise parce qu'elle (du moins 


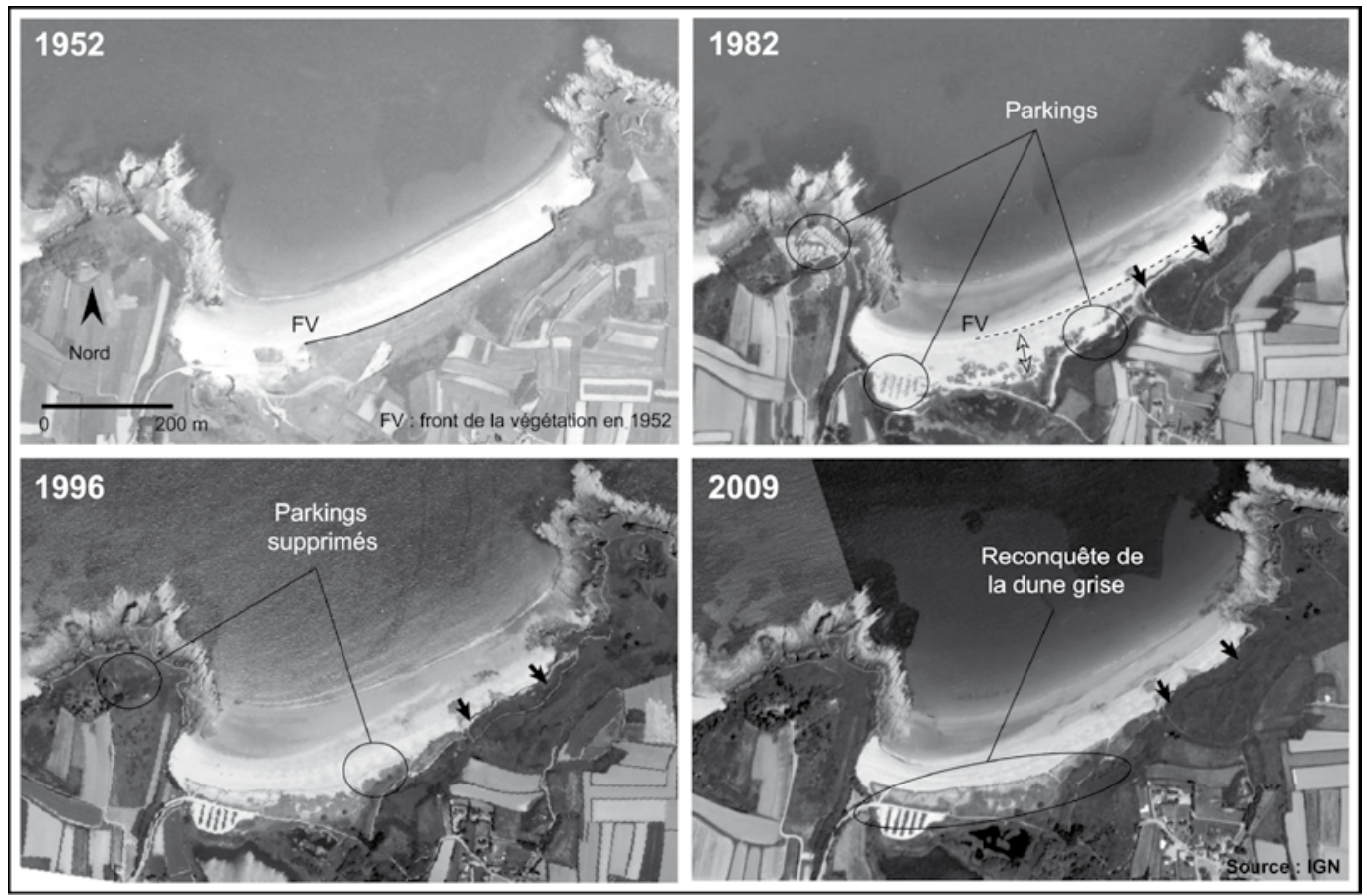

Figure 5 : Évolution du site d'après les photos IGN Evolution of the study site, based on IGN air photos

son futur emplacement, désigné par B sur la figure 1) est recouverte par une dune « grimpante » (dérivé du terme anglo-saxon climbing dune, terminologie que l'on retrouve par exemple chez R. Bruce et al., 1998 ou de B. Cagnoli et T. Ulrych, 2001) qui ne porte aucune trace d'érosion : sa couverture végétale est totalement homogène et dense (étape 1). En 1977 (étape 2), la falaise apparaît. La dune qui la recouvrait se scinde alors en deux entités : un dépôt au pied de la falaise, l'autre à son sommet, à la façon d'une dune perchée (étape 3). L'explication de ce changement morphologique est simple. Le sable de l'Anse du Verger a été prélevé et le sommet de la falaise est utilisée comme parking : les voitures sont garées jusqu'à son bord. En 1980, le recul est faible (inférieur à la marge d'erreur) mais les indentations du sommet de la falaise témoignent d'une extrême variabilité spatiale de ce processus. La photo de 1982 produit le même type d'information avec la même interprétation (étape 4). Le ralentissement de la vitesse de recul est très probablement lié au fait que la falaise est maintenant fermée aux automobiles (1985). De 1990 à 1996, la dune « grimpante » disparaît progressivement et le socle apparaît parfois entre la plage et le head. Le taux de recul reste faible, de l'ordre de 0,1 à $0,4 \mathrm{~m} / \mathrm{an}$ selon les endroits (étape 5 ). Il est presque entièrement produit par deux tempêtes coïncidant avec des vive-eau, celle de 1991 et celle de 1995 (Regnauld et Kuzucuoglu, 1992; Regnauld et al., 1998). De 1996 à 2008, le recul devient presque négligeable $(0,1$ à $0,2 \mathrm{~m} / \mathrm{an})$, jusqu'à la tempête de mars.

Durant la même période, l'évolution du cordon dunaire (en A sur la figure 1) comporte trois étapes principales que la figure 6 représente schématiquement. De 1948 à 1952, il y a accrétion et il semble que la végétation gagne vers le large (étape 1), mais le mouvement mesuré est inférieur à la marge d'erreur. La phase d'érosion est perceptible sur les photos de 1977, 1980 et 1982 mais elle a commencé en 1960 (prélèvements de sables pour l'usine de la Rance [étape 2]) et a duré jusqu'en 1985. En 1977, le cordon est considérablement érodé et sa couverture végétale est dis- 
continue (étape 3 sur la figure 6). En 1980 et 1985, la couverture végétale devient de plus en plus rare. La pose des ganivelles à partir 1985 (étapes 4 à 6 sur la figure 6) permet une reconstitution rapide. La photo de 1996 est un peu particulière car elle ne rend pas compte de cette reconstruction : elle présente l'état du système après la tempête de 1995 (étape 7 de la figure 6) : la ligne de ganivelles replacées post-tempête est bien visible mais la végétation n'a pas encore totalement re-colonisé les espaces que la tempête avait érodé, ou recouverts de washover. En 2000, le cordon a complètement retrouvé son apparence d'avant la tempête de 1995 (étape 8). Jusqu'en 2008, le système croît en hauteur (environ $0,25 \mathrm{~cm}$ d'altitude gagné chaque année derrière les ganivelles) et de nouvelles ganivelles sont posées tous les quatre ou cinq ans, un peu en avant des précédentes (vers le nord). Ce type de gestion basée sur l'utilisation de ganivelles fait qu'en 2008 le front du cordon a pratiquement retrouvé l'emplacement de 1948.

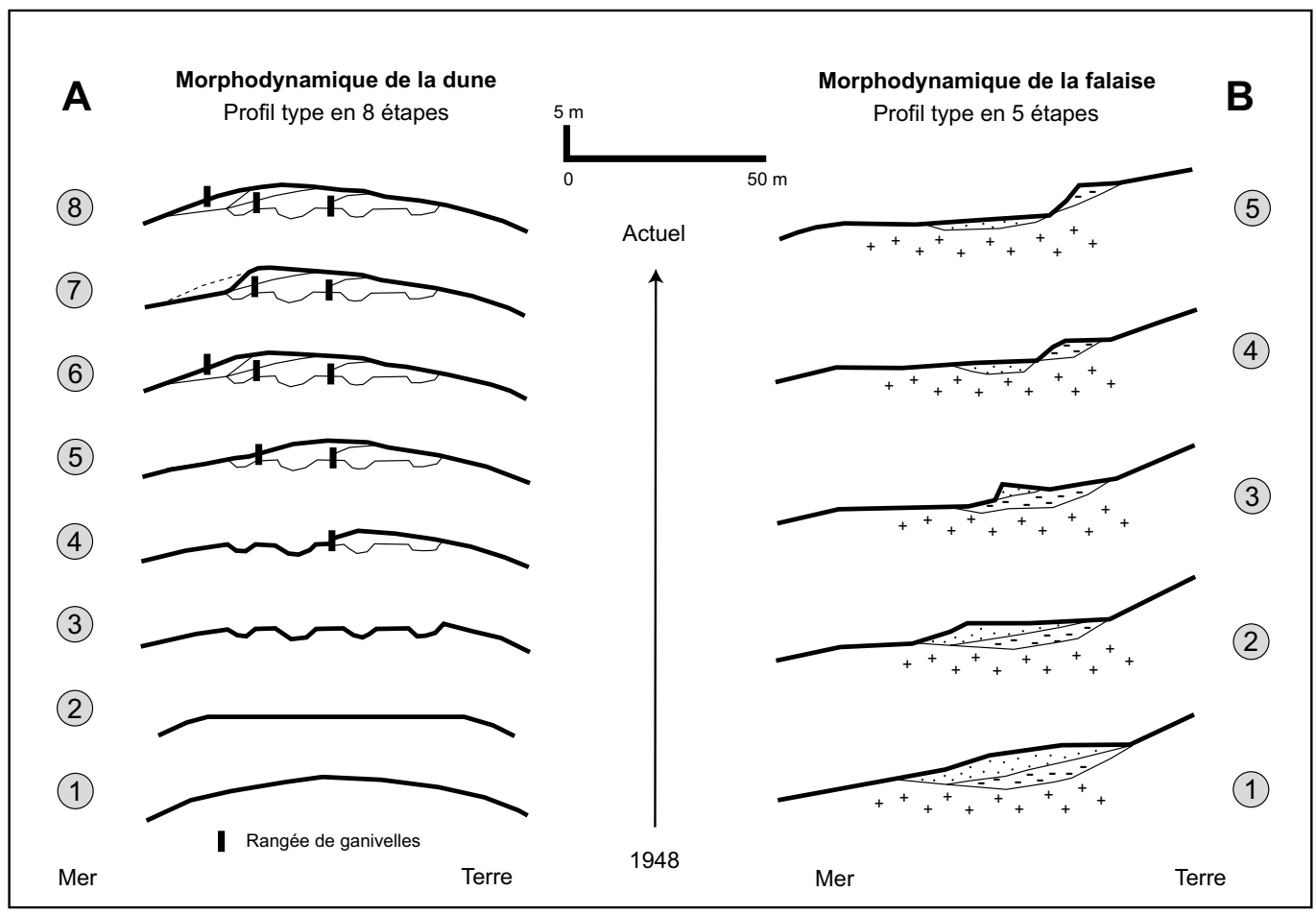

Figure 6 : Schéma synthétique de l'évolution de la dune (A) et de la falaise (B) établi avec les photos aériennes et les documents fournis par les aménageurs et le Conservatoire du Littoral (les croix signalent le socle, les tirets le matériel périglaciaire et les points le sable)

Synthetic sketch of the evolution of the dune (A) and of the clif (B)f. Data from air photos and from the Conservatoire du Littoral (crosses are for the in situ rocks, hyphen for periglacial material and dots for sand)

\section{Discussion : variabilité spatiale, variabilité dans le temps et variabilité des forçages}

La connaissance de l'évolution sur le moyen terme (60 ans) et du rôle de chacune des trois tempêtes (1991, 1995 et 2008) permet de réfléchir à quatre notions : 1) la variabilité des reculs, 2) le bilan sédimentaire d'un événement, 3) la vitesse d'évolution et des processus qui la contrôlent, 4) 
le concept d'accommodation space, qui s'avère très utile pour articuler ces différents éléments d'analyse.

Sur la falaise la vitesse de recul est variable d'une tempête à l'autre et d'une partie de la falaise à l'autre. Ces deux variabilités (spatiale et temporelle) sont liées à la nature et à l'épaisseur des strates qui composent l'escarpement. La figure 7 représente trois portions de la falaise (lignes). Les trois dates (colonne) correspondent aux situations immédiatement postérieures aux trois tempêtes majeures étudiées (1990, 1995 et 2008). La première a engendré un recul relativement uniforme sur toute la falaise. Pour la deuxième, c'est encore assez vrai, à ceci près toutefois que dans la partie ouest il est faible. Cela s'explique par le fait qu'à la base de cette portion de la falaise affleure un cordon de galets fossile (unité 2 sur la figure 2) et qu'en conséquence le pied de la falaise est plus résistant que là où il est composé de head, ou d'une dune ancienne. Il est aussi possible de constater une autre raison que la simple résistance mécanique : durant la tempête, le head se sature en eau alors que le cordon de galets fossile a une très forte porosité. Dans les jours qui suivent, le head saturé connaît des effondrements en masse alors que les galets ne bougent pas. Pour la troisième, le recul est extrêmement inégal suivant les lieux. En A, il est important mais semblable à celui des tempêtes précédentes. En B, il est considérable : le sommet de falaise recule localement de plus de 5 mètres en une seule journée. La base bouge très peu parce qu'elle est constituée par un affleurement in situ du socle migmatique, recouverts par des paquets effondrés (cartouche 7 , fig. 7). Le pied de la falaise est, à cette date, constitué par le socle qui a la forme d'un plan incliné. Il est donc probable que cette configuration géométrique ait permis aux houles d'atteindre une limite de run-up significativement plus élevé que dans le cas précédent, provoquant ainsi un fort recul du sommet. Enfin, en A, le cordon de galets fossile est en partie dispersé mais joue encore un peu son rôle de protection à la base du profil. Le recul est similaire à celui engendré par la tempête de 1996. Cependant, en janvier et février 2009, trois tempêtes d'intensité modérée ont presque entièrement fait disparaître ce qui restait du cordon de galets. La prochaine forte tempête aura probablement des effets légèrement différents. En $\mathrm{A}$, le recul commence à permettre au socle d'affleurer et il est possible que la vitesse de recul de la base diminue. Le sommet reculera sans doute encore vite, ne serait ce que par effondrement. En B, le matériel disponible pour le recul est presque entièrement érodé. Les reliques de matériel périglaciaire sont peu épaisses et peu étendues. Rien que par ruissellement durant un hiver pluvieux (2008-2009), l'essentiel de ce sédiment a été évacué vers le pied de falaise et incorporé à la plage. Le socle affleure quasiment partout et il est assez résistant pour ne plus reculer face à une tempête. Dans l'espace, cette portion de la côte va devenir stable. En C, le cordon de galet ayant quasiment disparu, il est probable que la vitesse de recul soit accrue. L'observation des effets des tempêtes doit donc conduire à une grande prudence quant aux extrapolations spatiales des phénomènes de recul.

Un second objet de discussion est celui du bilan sédimentaire. Selon les idées exposées dans l'introduction, le recul du site source doit alimenter le site puit. Sur le long terme (chronique de photos aériennes de 1980 à 2006), le recul de la falaise coïncide avec l'engraissement du cordon dunaire. Certains artéfacts arrachés à la falaise sont retrouvés dans le cordon : il y a bien transfert de matériel. Ce qui est moins clair est d'évaluer l'importance de cet apport latéral. On peut tenir pour certain qu'une part du matériel arraché à la falaise se retrouve sur la plage sous-marine, en particulier la fraction granulométrique comprise entre 1 et $0,25 \mathrm{~mm}$. Le matériel plus grossier reste au pied de la falaise ou sur le haut de plage. Le matériel plus fin est probablement dispersé plus au large. Il n'est pas évident de savoir comment le matériel qui a ainsi enrichi la plage sousmarine va migrer sur la plage puis sur le cordon. Un processus identifié est le dépôt par washover. Ce sont des apports spectaculaires mais limités en volume. Ils ne peuvent à eux seuls être tenus pour responsables de la croissance verticale du cordon dunaire derrière les ganivelles. Il est en revanche possible d'avancer qu'une part importante du sable que le vent souffle sur le cordon provienne, non pas de la falaise mais du large et soit déposé sur la plage lors de vives eaux calmes, puis ensuite mobilisé par le vent. En somme, il n'y a pas de lien causal suffisamment clair entre le recul de la falaise et la croissance du cordon dunaire pour établir absolument que l'Anse du 

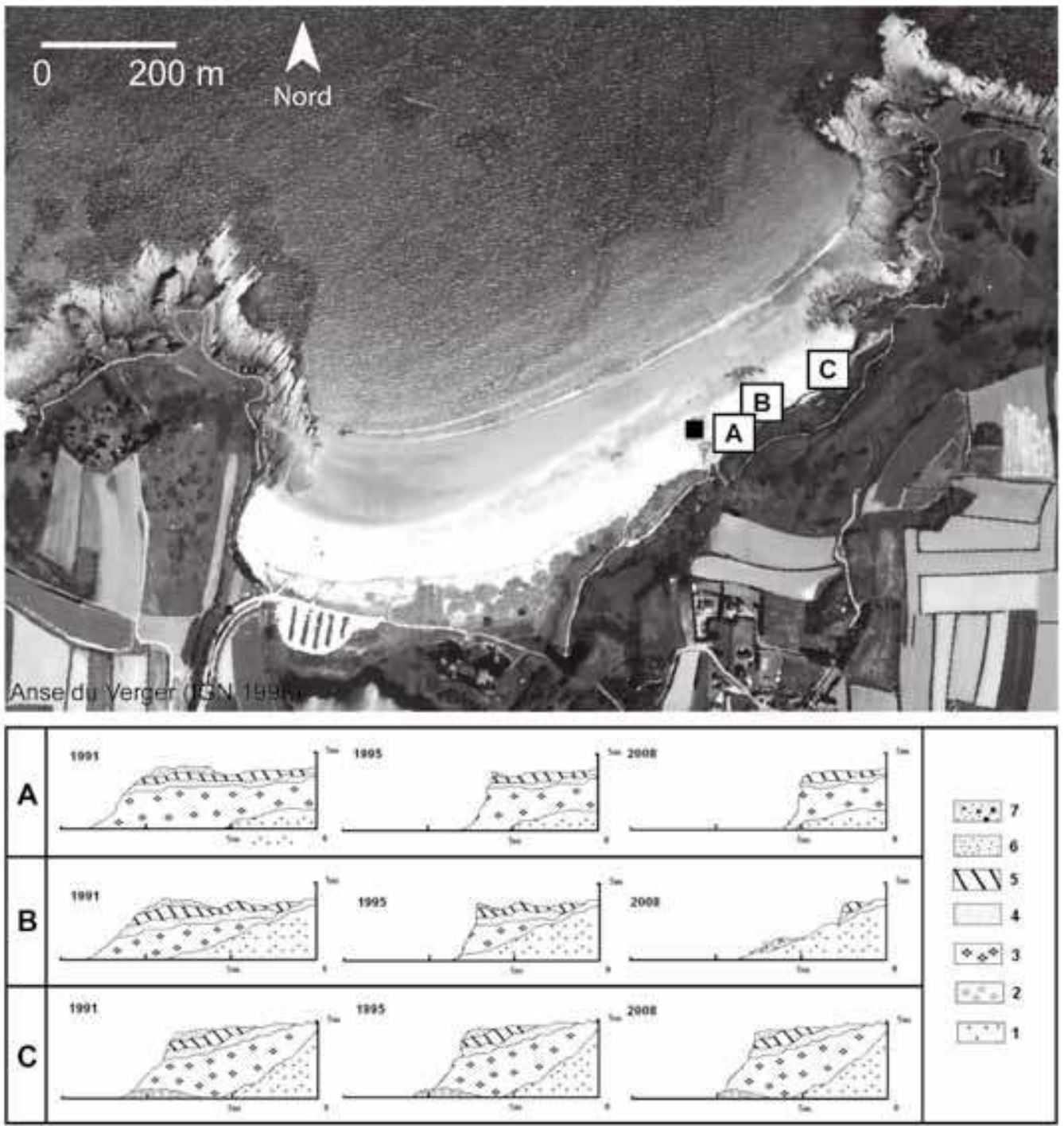

Figure 7 : Modalité du recul selon la structure sédimentaire de la coupe exposée aux houles 1 : Socle. 2 : Galets fossiles. 3 : Head. 4 : Loess. 5 : Dune et sols holocènes. 6 : Dune sub-actuelle. 7 : Matériel effondré.

Processes of retreat and exposures of sediment layers in the cliff face

1: Basement; 2: Fossil pebbles; 3: Head; 4: Loess; 5: Holocene dune and soils; 6: Sub-present dune; 7: Collapsed sediment.

Verger fonctionne comme une cellule sédimentaire simple. Il y a des transferts falaise-cordon mais il se peut qu'ils ne soient qu'anecdotiques et que l'essentiel des apports viennent du large. Si cette hypothèse est avérée, la définition des cellules sédimentaires comme unités spatiales de gestion de chaque baie doit être étendue vers le large jusqu’à la profondeur de clôture, ce qui demande que celle-ci soit connue.

Un troisième objet de discussion relève du comportement et des vitesses d'évolution. L'étude de l'évolution de ce site donne des informations intéressantes : en moyenne, de 1948 à 2008, le 
recul de la côte est de $1 \mathrm{~m} / \mathrm{an}$ dans la partie est de l'Anse, alors que dans la partie ouest, recul (de 1952 à 1985) et re-avancée (1985 à 2008) se compensent, si bien que et le trait de côte, géométriquement parlant, pourrait être considéré comme stable. Il suffirait de comparer la photo de 1948 et la topographie actuelle (2009), on pourrait conclure à l'immobilisme. Cependant cette «stabilité » n'a aucun sens morphologique. Les deux portions du littoral, qui sont spatialement voisines, exigent donc d'être comprises avec des schémas temporels distincts et incompatibles en apparence l'un avec l'autre. Pour la falaise (B sur la figure 1), l'ensemble du comportement morphologique est relativement simple à modéliser. Il y a un recul constant, qui est principalement dû à l'effet de fortes tempêtes et à leurs conséquences différées dans le temps (effondrements post-tempêtes). Pour le cordon dunaire (A sur la figure 1), le schéma est beaucoup moins simple. On peut dire que le forçage majeur est double : il y a à la fois fourniture de sédiment (ce qui réfère à l'extension spatiale de la zone d'approvisionnement) et fixation du sable par les ganivelles (ce qui renvoie à des décisions politiques). Suivant la nature des décisions prises par les responsables de l'aménagement, le cordon dunaire peut avoir tous les comportements possibles, du recul à la progradation. Si les « bonnes » pratiques d'aménagement, ganivelles et mise en réserve, sont mises en œuvre les épisodes de tempêtes ne sont que des événements perturbateurs dont les effets sont réversibles. Il y aurait donc une résilience entretenue socialement (au sens de C. Vogel et al, 2007) et pas absolument naturelle.

Enfin, le concept d'accommodation space est récent en géomorphologie. Il a été utilisé pour expliquer le fonctionnement géomorphologique d'estuaires micro tidaux (Anthony et al., 1996; Lang et al., 1995) mais il est encore assez peu employé en géographie et en aménagement (Baudelle et Regnauld, 2004). Il correspond à une étendue nécessaire au fonctionnement d’un système soumis à des forçages, météo-marins dans le cas qui nous intéresse. Il rassemble l'espace actuellement occupé par le système, celui qu'il a occupé et celui qu'il peut occuper : «The accommodation space includes the old space, which has not been filled in early period and new space, which is formed in the process » (Zaixing et al., 2008). Cette conception correspond assez étroitement à un concept qui avait été proposé par E. Lee (1998). Il proposait de lier explicitement le concept de cellule sédimentaire avec celui de recul du sommet de falaise au travers de la notion de "CBU » (cliff behaviour unit) : " the concept of cliff behaviour unit (CBU) provides an important framework: These units span the nearshore to the cliff top and are coupled to adjacent CBUs within the framework provided by littoral cells. » Le concept de cellule sédimentaire est alors utile mais il doit être entendu aussi au sens d'accommodation space et de CBU, c'est-à-dire d'espace d'où le sédiment provient et d'espace nécessaire à la mobilité des formes, y compris à leur recul suite à une tempête. Au Verger, l'accommodation space doit s'étendre au large (selon toute vraisemblance, jusqu'à la profondeur de fermeture) et doit comprendre le futur recul de la falaise. Il n'aurait donc pas été facile de le définir en 1948! Il est également nécessaire de prendre en compte la très forte variabilité temporelle des rythmes d'évolution, qui dépend d'une part de la fréquence des événements exceptionnels et d'autre part de la longueur des épisodes « calmes ». Enfin il faut considérer que les interventions anthropiques sont, la plupart du temps, les déterminants les plus importants pour « forcer » la forme à se reconstituer en un périmètre précis.

\section{Conclusion}

L'évolution du littoral est certainement liée aux tempêtes mais ce lien n'est pas univoque. Dans certains cas la tempête est l'agent principal, il fixe le sens de l'évolution (recul irrémédiable), ou encore le rythme des changements. Dans d'autres cas, la tempête n'est qu'un phénomène exceptionnel et l'évolution reste liée à la domination des conditions moyennes. Les deux points de vue sont vrais et ne sont pas inconciliables dans l'espace : ils concernent des portions de côtes adjacentes. Pour appréhender le comportement de ce type de littoral, il faut donc absolument prendre en compte une étendue spatiale qui dépasse l'extension de la simple «forme ». Ce travail a tenté de tester sur un exemple une conception théorique du littoral qui permette cette prise en 
compte. Il s'agirait de concevoir le comportement du littoral comme un tout et de penser, dans le cadre d'une cellule sédimentaire qui décrirait le fonctionnement «naturel », ou «normal »du littoral, à la fois : 1) le recul du site source et l'espace que ce recul concerne, 2) l'engraissement du site puits et l'espace nécessaire à sa croissance, 3) la variabilité des sites de transits et leur mobilité spatiale. L'idée est d'utiliser la notion de CBU comme l'équivalent, pour le site qui recule, de l'accommodation space pour l'accumulation sédimentaire. Si cette idée est pertinente, il est alors envisageable de tenter de définir quel est l'espace nécessaire au fonctionnement de l'ensemble d'une cellule sédimentaire et non pas se limiter à étudier celui qui est utile au fonctionnement des seuls sites puits. L'enjeu (soulevé par J. Hooke dans l'ensemble de son ouvrage de 1998, aussi bien que par D. E. Hart et K. R. Bryant en 2008) est de donner les mêmes lettres de noblesse à l'espace qui recule qu'à celui qui prograde, les deux étant également nécessaires au fonctionnement d'une cellule sédimentaire, donc à la mobilité naturelle d'un littoral. Cela revient évidemment à relativiser l'aspect spectaculaire de reculs produits par de fortes tempêtes et à réinterpréter leur responsabilité dans le fonctionnement « normal » de la mobilité du littoral.

\section{Bibliographie}

Anthony E. J., LANG J., OyÉdÉ L. M., 1996. Sedimentation in a tropical, microtidal, wave-dominated coastalplain estuary, Sedimentology, n 43 , p. 665-675.

Baudelle G., Regnauld H. 2004. Échelles et temporalités, Belin, 174 p.

Bray M. J, Carter D. J., Hooke J., 1995. Littoral cell definition and budget for central southern England. Journal of Coastal Research, vol. 11, n², p. 391-400.

Bruce R., White B. R., Tsoar H., 1998. Slope effect on saltation over a climbing sand dune, Geomorphology, vol. 22, n² 2, p. 159-180.

Cagnoli B., Ulrych T. J., 2001. Ground penetrating radar images of unexposed climbing dune-forms in the Ubehebe hydrovolcanic field (Death Valley, California), Journal of Volcanology and Geothermal Research, vol. $109, n^{\circ} 4$, p. 279-298.

Cariolet J.-M., Costa S., Caspar R., Ardhui F., Magne R., Goasguen G., Aspects météo-marins de la tempête du 10 mars 2008 en Atlantique et en Manche, Norois, ce volume.

Carter R. W. G., Orford J. D., 1993. The morphodynamics of coarse clastics beaches and barrier : a shortand long- term perspective, Journal of Coastal Research, Spe. Iss., $n^{\circ} 15$, p. 158-179.

Cocaign J.-Y., Bizien Jaglin C., Regnauld H., 1996. Les sites protohistoriques de l'Anse du Verger en Cancale dans leur cadre archéologique et environnemental, Les cahiers de CeRAA, n 24, p. 71-84.

Cooper J. A. G., 2002. The role of extreme floods in estuary-coastal behaviour : contrasts between river- and tide- dominated micro tidal estuaries, Sedimentary Geology, vol.150, nº 1, p. 123-137.

Dodd J., Blondeaux P., Danie Calvete D., Huib E., De Swart G., 2003. Understanding Coastal Morphodynamics Using Stability Methods, Journal of Coastal Research, vol. 19, n 4, p. 849-865.

Dubois R. N., 1995. The transgressive barrier model: an alternative to two dimensional volume balanced models, Journal of Coastal Research, vol. 11, n 4, p. 272-1286.

Forbes D. L., Orford J. D., Carter R. W. G., Shaw J., Jennings S. C., 1995. Morphodynamic evolution, self organisation, and instability of coarse clastic barriers on paraglacial coasts, Marine Geology, vol.126, $\mathrm{n}^{\circ} 1-4$, p. 63-85.

Hart D. E., Bryant K. R., 2008. New Zealand coastal systems boundaries, connections and management, New Zealand Geographer, vol. 64, n 2, p. 129-143.

Hooke J., 1998. Coastal Defence and Earth Sciences Conservation, The Geological Society, London, 270 p.

Jervey M. T., 1988. Quantitative geological modeling of siliciclastic rock sequence and their seismic expression, dans Wilgus C. K., Histings B. S., Kendall C. A., et al., Sea level changes: an integrated approach. SEPM Special Publication n ${ }^{4}$ 42, p. 47-69.

LAng J., Anthony E. J., OyédÉ L. M., 1995. Late Quaternary coastal sediments in Benin: a preliminary sequence-stratigraphic interpretation. Quaternary International, n² 29/30, p. 31-39. 
LEE E., 1998. Problems associated with the prediction of cliff recession rates for coastal defence and conservation, dans Hooke J. (dir.), Coastal Defence and Earth Sciences Conservation, The Geological Society, London, p. 46-57.

Musereau J., Regnauld H., Planchon O., 2007. Vulnérabilité aux tempêtes des dunes littorales : développement d'un modèle de prédiction du dommage à travers l'exemple de Saint Trojan (île d'Oléron, France), Annales de l'Association Internationale de Climatologie, vol. 4, p. 1-22.

Orford J. D., Forbes D. L., Jennings S. C., 2002. Organisational controls, typologies and time scales of paraglacial gravel-dominated coastal systems, Geomorphology, vol. 48, n 1-3, p. 51-85.

Regnauld H., Kuzucuoglu C., 1992. Rebuilding of a dune field landscape after a catastrophic storm: beaches of Ille-et-Vilaine, Brittany, France, dans Carter R. W. G., Curtis T. G. S., Sheedy-Skeffington M. J. eds., Coastal Dunes, geomorphology, ecology and management for conservation, Balkema, Rotterdam, p. $379-387$.

Regnauld H., Cocaign J.-Y., Salièges J.-F., Fournier J., 1995. Mise en évidence d'une continuité temporelle dans la constitution de massifs dunaires du Sub Boréal (3600BP) à l'Actuel sur le littoral septentrional de la Bretagne. Un exemple dans l'Anse du Verger (Ille-et-Vilaine), Comptes Rendus de l'Académie des Sciences, vol. 321, n 4, p. 303-310.

Regnauld H., Jennings S., Delaney C., Lemasson L., 1996. Holocene sea level variations and geomorphological response: an exemple in Northern Brittany, Quaternary Science Reviews, n 15, p. 781-787.

Regnauld H., Fournier J., Gouéry P., 1997. Geomorphology and submarine landscapes of rocky platforms preeceding cliffs in Brittany (France), Memorie descriptive carta geologica d'Italia, vol. LII, p. 179-189.

Regnauld H., Lemasson L., Dubreuil V., 1998. The mobility of coastal landforms under climatic changes: issue for geomorphological and archeological conservation, dans Hооке J. (dir.), Coastal Defence and Earth Sciences Conservation, Geological Society Publishing House, p. 103-114.

Regnauld H., Mauz B., Morzadec-Kerfourn M. T., 2003. Shorelines of the last interglacial in western France: some preliminary results with OSL datings, Marine Geology, vol.194, nº 1-2, p. 65-77.

Robin M., 2002. Télédétection et modélisation du trait de côte et de sa cinématique. dans Baron-Yellés N., Goeldner-Gianella L., Velut S. (dir.), Le Littoral : Regards, Pratiques et Savoirs : études offertes à F. Verger, Presses de l'Ecole Nationale Supérieure, Paris, p. 95-115.

Roy P. S., Cowell P. J., 1996. Simulation Modelling of Large Scale Coastal Behaviour: A Review. IGCP 367 Annual Meeting, Late Quaternary Coastal Records of Rapid Change: Applications to present and future conditions, Sydney, p. 1-16.

Pian S., Regnauld H., Menier D., 2008. Interactions nature/société et vulnérabilité du littoral : quelques exemples en Bretagne Sud. Actes du colloque international pluridisciplinaire « le littoral : subir, dire, agir » [http://www.ifresi.univ-lille1.fr/Littoral2008/Themes/Theme_4/Seance1/Pian. pdf].

Vogel C., Moser S. C., Kasperson R. E., Dabelko G. D., 2007. Linking vulnerability, adaptation and resilience science to practice: pathways, players and partnerships. Global Environment Change, vol. 17, $n^{\circ} 3-4$, p. 349-364.

Zaixing J., Yingchang C., Weili Y., Tong W., Le Z., 2008. Accommodation Space Transformation System in Faulted Basin. The Open Geology Journal, vol. 2, p. 9-17.

Cet article a été reçu le 22 septembre 2009 et définitivement accepté le 24 mars 2010. 